\title{
Research on Community Grid Management System Based on 3D GIS
}

\author{
Yajing Liu*, Jiming Yao, Jing Shi \\ College of mining engineering,North China University of Science and Technology,Tangshan 063009,Hebei, \\ China. \\ * Corresponding author. Tel.: 5713635962; email: lyj2206@163.com \\ Manuscript submitted June 10, 2016; accepted: August 13, 2016 \\ doi: $10.17706 /$ jsw.11.10.1026-1039
}

\begin{abstract}
Since the traditional community management mode and method have been unable to adapt to the pace of urbanization process, the urban grid management model becomes the trend of community management. The community environment is shown in the form of two-dimensional maps in traditional community information platform, which is not intuitive and not realistic. The three-dimensional display can both express the plane and vertical relationship among community management objects, which is more intuitive, thus creating a vivid three-dimensional interactive scenery can facilitate the management of community. The grid management model of the experimental community is designed in this paper. We build the model of the terrain and objects which are classified in the community, design and develop a grid community management system. The system realizes the integration of the resources in the community. The management process has been gradually realized in the system, which meets the management requirement of informational and integrative, makes the community management services more precise and efficient, and provides a new way for urban community management.
\end{abstract}

Key words: 3D GIS, three-dimensional object model, grid management, system architecture.

\section{Introduction}

Since "Digital and Comfort Community Construction ",that is "Digital City" was formally proposed initiative by AI.Core in September 1998,the United States launched a digital construction, and built a number of "demonstration project of Intelligent living area digital community" ,for example ,the Washington Community Service Network, which can provide residents with a variety of services . In addition, the "smart city " concept was first proposed by the Singapore Government , and as early as 1996 ,the "One Project" began to be implemented in Singapore, and the E-civic center . was established in April 1999.Moreover, the government spent \$ 1.5 billion in the year 2005 to build E-Government, E-Government can allow citizens to conduct the business online, and even provide services related to the query for travelling to domestic citizens and government departments [1], Japan is not only built a demonstration project which is "Smart Living Area Digital Community ", Kyoto University and Japan Telecom develop "Virtual Kyoto ", and the most achievement of intelligence community of Japanese is the "Electronic Cottage " in 2010,it is popular basically in the whole society [2]-[4]. The intelligence community of Japanese pay more attention to meet the needs of residents , and achieve high efficiency and all-round service through the establishment of local area network. Through the study of the present situation of the foreign community digitalization, the construction of community information database, connectivity of the network situation, and the realization of a number of Internet and electronic 
community service of these countries have reached the advanced level, and fully embodied in the people-oriented, so the foreign successful technology and experience is worth our reference. China's digital city is the focus of the national information construction, with the development of digital city, digital community research is developing fast, although the development is a shorter time, but it is faster. The government attaches great importance to the construction of digital city and community informatization, many cities such as Shanghai, Guangzhou, Hangzhou, Xiamen and other large and medium cities have developed a corresponding plan to build the digital city, and some cities have also built a smart district or smart home test project [5].The research on the digital management of the community is still in the development of some technical systems in China, and the research on the change of the community management system is lack. Therefore, it is of great significance to pay attention to the theoretical research of the community management system, and combine the theory research with the digital management technology to realize the real technical service management. It is an important to create a model different from the previous administration to promote the development of community management.

In recent years, with the acceleration of the urbanization process and the improvement of people's living standard, the management and development of urban communities are facing new problems and challenges. Because the community has the characteristics that whose population is large and whose daily affairs are various, and the community management departments are complicated and whose function is not clear, the issues that the responsibility cannot be implemented, the transaction period is long, the effect is not good, the management department and the relevant functional departments obtain information obviously lagging. These problems reflect the traditional management model has been unable to meet the needs of urban community management in the new situation. There is an urgent need to create a new model which is different from the traditional management pattern. There is an urgent need to form advanced management concepts and methods to promote the development of community management work. The community grid management model provides a new way of community management. But from a technology perspective, it is the realization of the two-dimensional grid electronic map view and can provide the plane information to the user. The form is lack of effective 3D space expression ability, cannot display 3D real situation of community buildings and facilities, making the management managers have great cognitive limitations to the community environment, so as to make the management behavior has certain limitation [6].

The paper takes a new core residential area of the city as the experimental area. The region occupies a total area of 494 acres, construction area of 660000 square meters, exists residents 6358 households, about 2 million people, which covers the largest area and where the largest number of population, and it is currently the largest dilapidated cottage resettlement area. The original 19 village residents move to this community. The location of the residential buildings is already rather different from the original. The original management departments of the village do not exist. Different from the old management model, to improve the community service and management function, a new community management model need be established.

This paper focuses on the construction of 3D community grid management system. On the basis of comprehensive analysis of the patterns and the research results of community grid management existing at home and abroad, according to the practical needs of the community management, relying on theory and technology of geographic information system (GIS) and 3D modeling, we build 3D community grid management system. The system displays the community environment in the form of three-dimensional and makes users query the information of the grid, residential buildings, population and facilities from real 3D quickly. The system realizes Case acceptance, Video surveillance and Comprehensive evaluation, making the management more informationalized and efficient [7]-[10].

\section{The establishment of Community Grid Management Model in the Experimentation Area}

Experimentation area community has the first, second, third, fourth communities, each community set up a neighborhood committee. To establish community grid management, we should detailed analyze five aspects 
which are the grid partition, management object classification, management personnel, management process and evaluation system. In order to offer basis for community gridded management system of the attribute data and spatial data organization, function design of the system.

\subsection{Grid Community Division}

According to the research on the community grid management mode, to realize the community grid management, we should divide the community into grid firstly. According to the unit grid management method, we should pay attention to some key factors, such as the administrative area boundary, the convenient management principle, the unit grid division principle and so on [11].

The administrative boundary element is that the boundary of the community, which is the largest boundary of the cell grid, and the unit grid cannot exceed the scope of the community boundary, so dividing the unit grid is the basis and important step. On the basic of surveying and mapping of 1:500 topographic map, graph corresponding to the community boundaries is drawn, and it is used as a grid based map, grid partition should be in the boundary of courtyards, districts, streets and alleys. According to the basic principle of dividing grid unit, arranged the number of 300 to 500 households, population about 1000 to 1500 people as a grid, 30 to 50 families set up a group of buildings, horizontal to edge, vertical in the end, grid without leaving gaps in the management and grid does not cross, overlap, area in all primary and secondary roads, alleys, public places, residential buildings, enterprises and institutions, such as into grid. The experimentation area is divided into 19 grid units, in which the first community is divided into 5 grids, the second community is divided into 6 grids, the third community is divided into 5 grids, and the fourth community is divided into 3 grids. The details of each grid are shown in Table I.

Table 1. Community Grid Situation

\begin{tabular}{|c|c|c|c|c|c|}
\hline Community & Grid & Number & The No. of building & Households & population \\
\hline \multirow{5}{*}{ first } & 1 & 3 & 304、305、307 & 327 & 1000 \\
\hline & 2 & 5 & $115 、 116 、 117 、 301,302$ & 410 & 1500 \\
\hline & 3 & 6 & $\begin{array}{l}\text { 107、108、109、111、112、 } \\
113\end{array}$ & 361 & 1150 \\
\hline & 4 & 2 & $104 、 105$ & 345 & 1100 \\
\hline & 5 & 2 & 101、102 & 345 & 1050 \\
\hline \multirow[t]{7}{*}{ second } & 6 & 3 & $303 、 306 、 308$ & 275 & 1150 \\
\hline & 7 & 3 & 110、114、118 & 326 & 1000 \\
\hline & 8 & 2 & 103、106 & 326 & 1000 \\
\hline & 9 & 3 & $401 、 404 、 408$ & 275 & 950 \\
\hline & 10 & 3 & 208、209、210 & 326 & 1050 \\
\hline & 11 & 2 & 201、205 & 368 & 1150 \\
\hline & 17 & 5 & $211,212 、 213 、 214,215$ & 410 & 1350 \\
\hline \multirow[t]{4}{*}{ third } & 18 & 2 & 202、206 & 418 & 1400 \\
\hline & 19 & 3 & 203、204、207 & 483 & 1500 \\
\hline & 12 & 1 & 415 & 210 & 750 \\
\hline & 13 & 2 & 411、 414 & 403 & 1300 \\
\hline \multirow[t]{3}{*}{ forth } & 14 & 3 & 410、412、413 & 267 & 1000 \\
\hline & 15 & 3 & 403、406、407 & 253 & 900 \\
\hline & 16 & 3 & 402、405、409 & 303 & 1100 \\
\hline total & & 56 & & 6431 & 21400 \\
\hline
\end{tabular}

\subsection{The Classification of Grid Management Objects in Experimental Community}

The management objects of the community can be classified into six categories: "people, buildings, matters, unit stores, events, organizations". The management of people and buildings can refer to the principle that is 
house manages people, and it can make the housing information and population information be contacted together. The matters are mainly refer to the various community components, such as all kinds of covers, road lamps, postboxes, dustbin, parking, bus stop and public facilities. According to "Urban municipal supervision and management information system-Classification, Coding and Data requirements for urban managed components and events" which is approved by the Ministry of Construction, all kinds of components are divided into six categories, which include public facilities, road traffic, city environment, garden greening, housing and land class and other facilities. Then we can do further small division on the basis of six categories [12]. About coding for components, the documents which are approved by the Ministry of Construction require that: the code of the components can be composed of 10 digits, the beginning six digits refer to the county-level administrative region code, the 7, 8 digits refer to the code of big categories, the final two digits refer to the code of small categories. According to the survey on the components of experimental community and the existing 1:500 topographic map data, classification and coding for the community components are as follows:

Table 2. Community Components Classification Code Table

\begin{tabular}{|c|c|c|c|}
\hline Big categories coding & Big categories name & $\begin{array}{l}\text { Small categories } \\
\text { coding }\end{array}$ & $\begin{array}{l}\text { Small categories } \\
\text { name }\end{array}$ \\
\hline \multirow{14}{*}{01} & \multirow{14}{*}{ public Utilities } & 01 & Supply water cover \\
\hline & & 02 & Polluted water cover \\
\hline & & 03 & Rainwater cover \\
\hline & & 04 & Rainwater comb cover \\
\hline & & 05 & Electricity cover \\
\hline & & 07 & Communication cover \\
\hline & & 10 & Heat cover \\
\hline & & 11 & Gas cover \\
\hline & & 13 & Firefight facilities \\
\hline & & 16 & Electricity facilities \\
\hline & & 18 & Lights \\
\hline & & 26 & Fitness facilities \\
\hline & & 34 & Transformer(box) \\
\hline & & 36 & Monitoring facilities \\
\hline \multirow{4}{*}{02} & \multirow{4}{*}{ Road traffic } & 01 & Parking \\
\hline & & 03 & Bus stop kiosks \\
\hline & & 04 & Taxi stop sign \\
\hline & & 10 & Traffic light \\
\hline \multirow{3}{*}{03} & \multirow{3}{*}{ City environment } & 01 & Public toilets \\
\hline & & 05 & Trash \\
\hline & & 07 & billboard \\
\hline \multirow{4}{*}{04} & \multirow{4}{*}{ Greenland } & 02 & Street tree \\
\hline & & 05 & Greenbelt \\
\hline & & 06 & sculpture \\
\hline & & 07 & Street Seats \\
\hline \multirow{3}{*}{05} & \multirow{3}{*}{ Housing land } & 01 & Propaganda column \\
\hline & & 02 & defense fortifications \\
\hline & & 03 & basement \\
\hline
\end{tabular}

The important function in the grid management system is the management of grid components. According to the above components classification, the corresponding three-dimensional models are established according to the division of small categories. The models are taken as the data base of the system, which make the system achieving visualization of grid components, components information query and component issue report.

\subsection{The Function Division and Evaluation System of Experimental Community Grid Management}

Experimental community belongs to a street administratively. The upper level administrative department sets up the social service management and integrated command center which is within the scope of jurisdiction, and 
each street social service management center and social services workstation tertiary work platform which are for each community, four interconnected of the District, street, community and the grid are realized. The community sets first, second, third and four communities. Each community has community service management workstation, a instructor, a police officer, a judicial officer and a inspector. Each grid in the community sets a full-time administrator, an assistant, a secretary of Party branch of each one. The Organizational Structure setting is shown in Fig. 1.

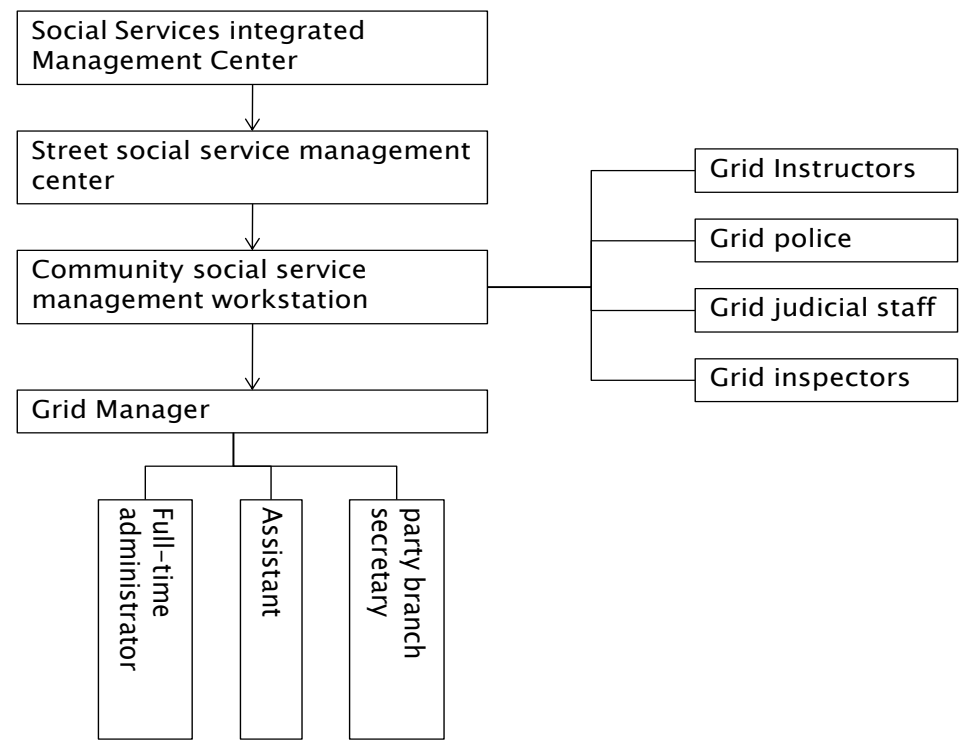

Fig. 3. Management organization and personnel settings.

On the basis of making clear the specific duties and detailed division of work of the managers, we reestablish workflow of experimental community grid management. The specific process is divided into seven steps: information collection, build files, task dispatch, field processing, feedback processing and verify, comprehensive evaluation, which form a complete closed-loop process [13], [14].

The grid management evaluation system of experimental community absorbs the excellent experience of the grid management of the national demonstration area, which mainly includes two aspects: external evaluation and internal evaluation. External evaluation mainly includes the evaluation of community public and media; internal evaluation mainly includes the evaluation information of grid management which is given by all levels of management center according to the relevant records of the grid management system and the actual situation.

\section{3D Terrain Model of the Community}

The geographical objects that constitute the three-dimensional landscape of the community are divided into two categories according to their spatial distribution characteristics: the terrain model and the object model. Terrain model is true expression of the ups and downs of the ground and the topography. These objects exist in the form of independent individual, and their types and shapes are various, so the modeling of independent features is with a certain complexity, does not have unified modeling method. Even so, the independent features of the community can be divided into some common types according to the characteristics. The 3D terrain models take discrete entities as objects, including buildings, street trees, dustbin, billboards and so on. The building is the most important type, and the appearance of the building is the myriads of changes, but according to the structure, buildings can be divided into three parts: the main part, part of the roof and accessory building [14]. In this paper, the object will be divided into four categories: ground vegetation, buildings, roads, public facilities. We choose the best model construction method respectively. The modeling processes of four kinds of different types of 3D objects are introduced as follows.

\subsection{Construction of three Dimensional Models of Community Buildings}


Residential buildings and related ancillary buildings are the main buildings of the community. The building structure is more complex. According to the types and characteristics of community buildings, we choose 3DMAX for building fine modeling.

Residential buildings are the important part of community three-dimensional landscape. Through the analysis of the structure pattern of residential buildings, residential buildings will be roughly divided into 9 categories, for each type of buildings using 3DMAX software modeling. The modeling process is divided into four parts: CAD drawings import, 3D modeling, texture mapping, models export.

1) CAD drawings import: the construction of models take CAD format topographic map as the base map. Since the layers of topographic map are messy and variety, so the other layers which have nothing to do with modeling need to be removed in order to ensure the conciseness and integrity of base map. When completing CAD map processing, before the map being imported into 3DMAX, first the unit of 3DMAX should be set mm, making the system unit scale and display units in the same proportion. Then the CAD file should be imported into 3DMAX, check the "re zoom" in the import Options dialog box, set the incoming file unit to $\mathrm{mm}$, and the other options are not changed. After importing files, select all objects as one group, and $\mathrm{x}, \mathrm{y}, \mathrm{z}$ axis as zero, and right-click to freeze the object. The map is taken as a base map, as shown in Fig. 2.

2) 3D modeling: before constructing 3D models of buildings, first we analyze the structure and appearance of the building, cutting the main building into simple geometry models in order to facilitate the construction and reduce the data of models. Next in the modeling process, some unnecessary details can be omitted, and the local appearance of the characteristic can be marked.

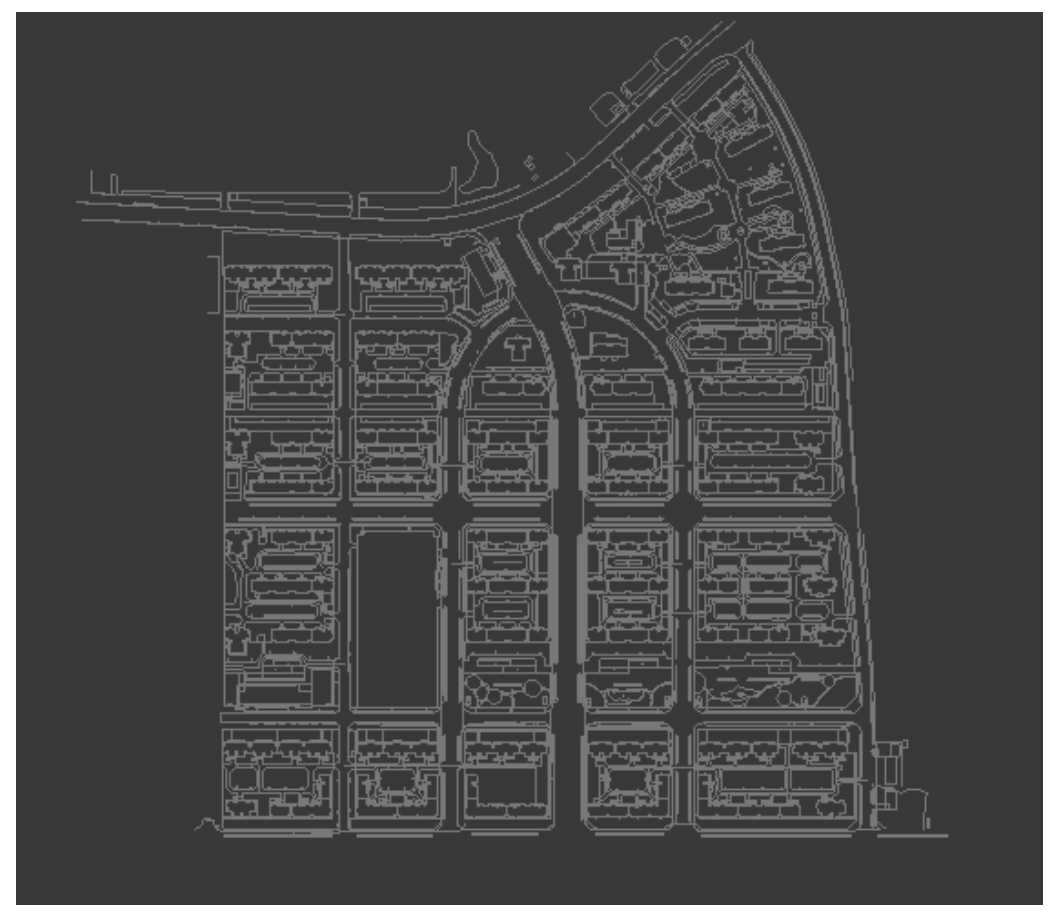

Fig. 4. Base map for modeling.

3) Texture mapping: first the texture mapping data which is dealt with assigned to the material, click on the material editor, click the "diffuse" pop-up "material / map browser", select "bitmap" pop-up "select the bitmap image file" window, select the image map, that the completion of the material given to. Next, select the material editor window "viewport display shading material" and "show the final results, click Assign materials to the selected object, can be the material attached to the specified object. The selected object, click on the "Edit" "UVW map" to adjust the 2D map coordinates, the establishment of the corresponding relationship between the object and the coordinates of the texture space coordinates, to ensure the correctness of the surface mapping and texture data, the mapping effect is more delicate, increase the realism of the model building. 


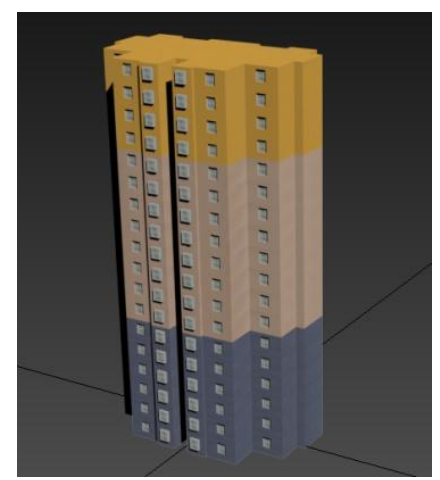

Fig. 5. Effect picture of the building.

4) Models export: finally, the constructed models of the residential buildings are derived .3ds format for the recognition of the ArcGIS, so that the models can be imported into the GIS software to carry out the integration of three-dimensional scene, as shown in Fig. 5.

\subsection{Construction of Vegetation and Roads}

The vegetation in the community is mainly planar grass, for the vegetation construction, other data should be deleted, leaving only a green layer which is CAD format to be imported into 3DMAX. According to the scope of green, we sketch the outline, extrusion molding, add the handled grass picture to the material to make it attached to the green object, and use the UVW map to adjust the texture coordinates, complete vegetation construction.

The road, traffic barrier, fence and so on in the community are all linear elements. The construction process of these elements, first of all, are expanding its form in the 3DMAX, and then making the texture of the texture production, so that a very high simulation.

\subsection{The Modeling of Affiliated Public Facilities}

In the community environment, lights, traffic lights, street trees, garbage cans and bus stop are community affiliated and public facilities, and which are the indispensable part. The street lights, trash and bus stop have regular geometry. For these regular models, ArcGIS software comes with corresponding symbol of 3D model, we take 2D vector map as the basis and give the corresponding features 3D symbol. But for traffic lights, trees and others whose shapes are more complex, it is more difficult to build their models. There are many ways to make the trees using 3DMAX, such as textures mapping. We can give diffuse and opaque texture to constructing objects. This method is simple and easy to operate. The system uses this method to establish trees models.

\subsection{The Integration of Community 3D Landscape}

Community three-dimensional landscape is integrated from the terrain model and object model in the ArcScene to achieve the realistic display of the community environment. The terrain model consists of the TIN model and remote sensing image data. We add TIN and remote sensing image data into the ArcScene. The hypsography of terrain is displayed in 3D scene through overlaying two kinds of data. The overlaying mentioned here is the value of the surface model in the area of the community as the elevation value of all kinds of factors in the scene. The three-dimensional display of the terrain in the 3D scene of the community is realized by taking the value of TIN model as the elevation value of the remote sensing image. Community three-dimensional landscape is shown in Fig. 6.

Each type of object in community has a corresponding two-dimensional point, linear or planar layer, 3D terrain model in ArcScene show that mainly by changing the point, the linear or planar layer data symbol attribute (symbol selector) [15]. Terrain 3D model which is made in 3DMAX software have been exported to.3ds format, the model need to be imported into ArcScene. First, all terrain model corresponding to the two-dimensional point, line or surface layer are loaded into the ArcScene. Then a three-dimensional display of these points, the linear or planar layer data: 1) data: the main buildings, trees, lights and so on. We has been built 
the object model and derive success in advance, then the three-dimensional model is imported symbol library, when the model is displayed as 3D symbol form in the scene, the right size can be gotten by changing the dimensions or attributes change this situation. And symbols are assigned to corresponding layer and adjusted until reach the realistic display effect, if the model is imported into ArcScene there are the actual deviation, we can choose 3D placement: by changing the normalized origin offset in $X, Y, Z$ values for small modifications, by changing the offset in $\mathrm{X}, \mathrm{y}$ and $\mathrm{Z}$ values of broad changes. Since we use the 3DMAX to build the model does not consider the model in the actual scene in a north-south direction, so after importing the scene may appear in the wrong direction. By changing the angle of the symbol (symbol selector attribute (angle) to adjust the model of direction. 2) data line: through the 3D map symbols of linear data display, mainly walls, billboards, double yellow lines, etc., for relatively simple lines show in this not to do too much introduction, mainly against the wall, bulletin board introduced. With the operation on the data points, the symbol attribute pop-up dialog box to choose 3D texture line symbol. The picture is displayed in the form of vertical by selecting vertical orientation, that is, the completion of construction of walls or billboard. If the width or height is not appropriate, you can adjust the Width. 3 surface data: mainly through the external graphics to display such as roads, green, turf, just like the surface data. In addition, you can change the Angle, ScaleX, or ScaleY properties to change the density or filling direction, and adjust the property until it reaches a good result.

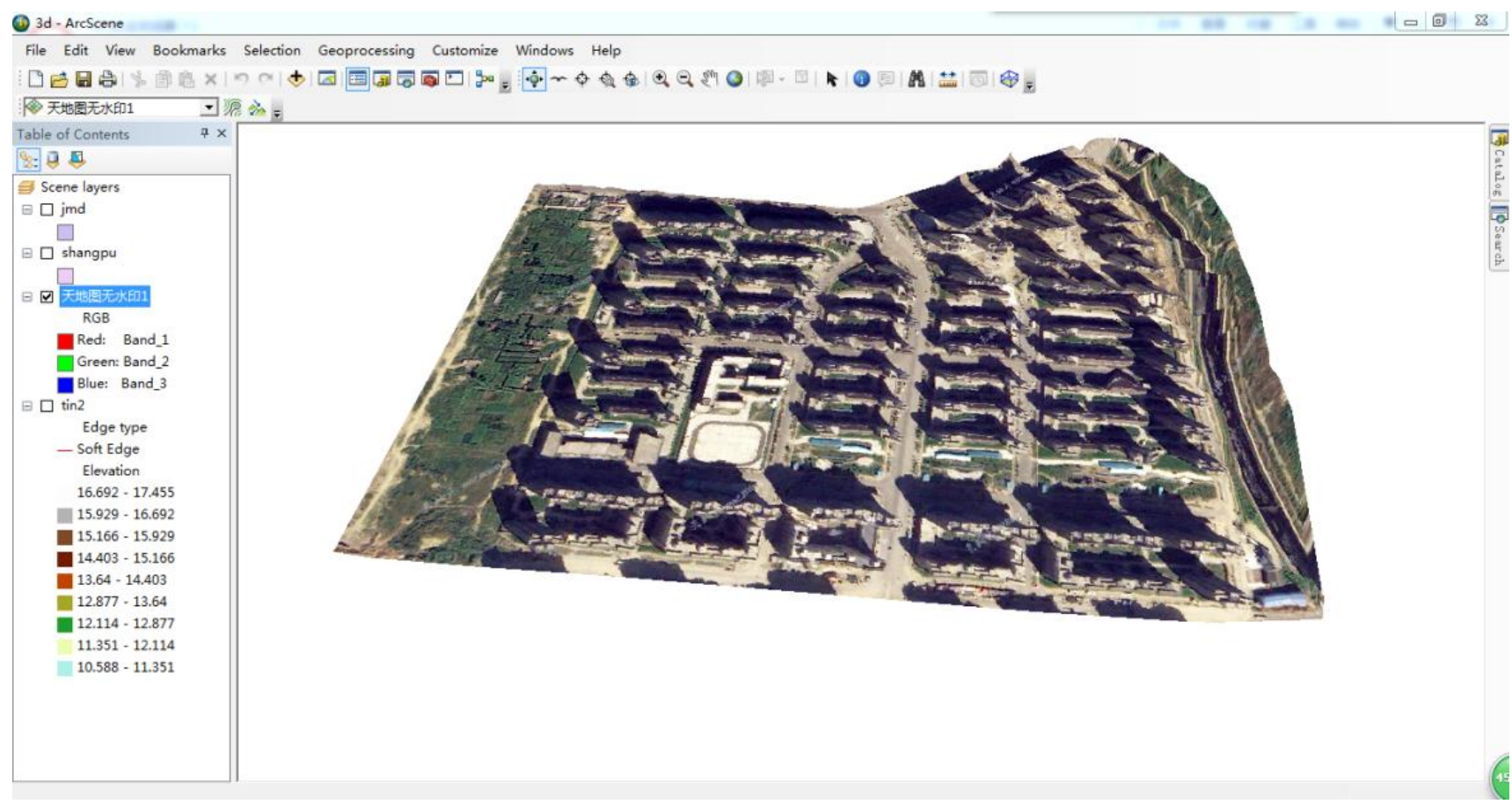

Fig. 6. Community terrain model.

The terrain model which consists of TIN and image data, and the 3D object model which consists of buildings, vegetation, street lamps and others are loaded into ArcScene. After adjusting the sign, scene optimization, three-dimensional landscape of the community is formed. The community 3D effect graph is shown in Figure 5.

\section{System Design and Implementation}

\subsection{System Architecture Design}

The system adopts three layer structure mode, from top to bottom: performance layer, application layer, data layer. The bottom layer provides service for the top layer. The system detailed structure is shown in figure 5 .

1)The data layer is taken as the base layer, which is responsible for storing and managing the spatial data and business data of the system, including two levels: the storage layer and the access layer. Storage layer stores 
spatial data and business data. The spatial data includes residents, green, water, transportation and other basic geographic data, and community components, community, grid and other thematic data. The business data is mainly community population, facilities, and events. Data access layer is the foundation of supporting the professional application services, through the ADO. Net provides attribute data access support based on two-dimensional relational tables for management, through the spatial data engine ArcSDE to spatial data access.

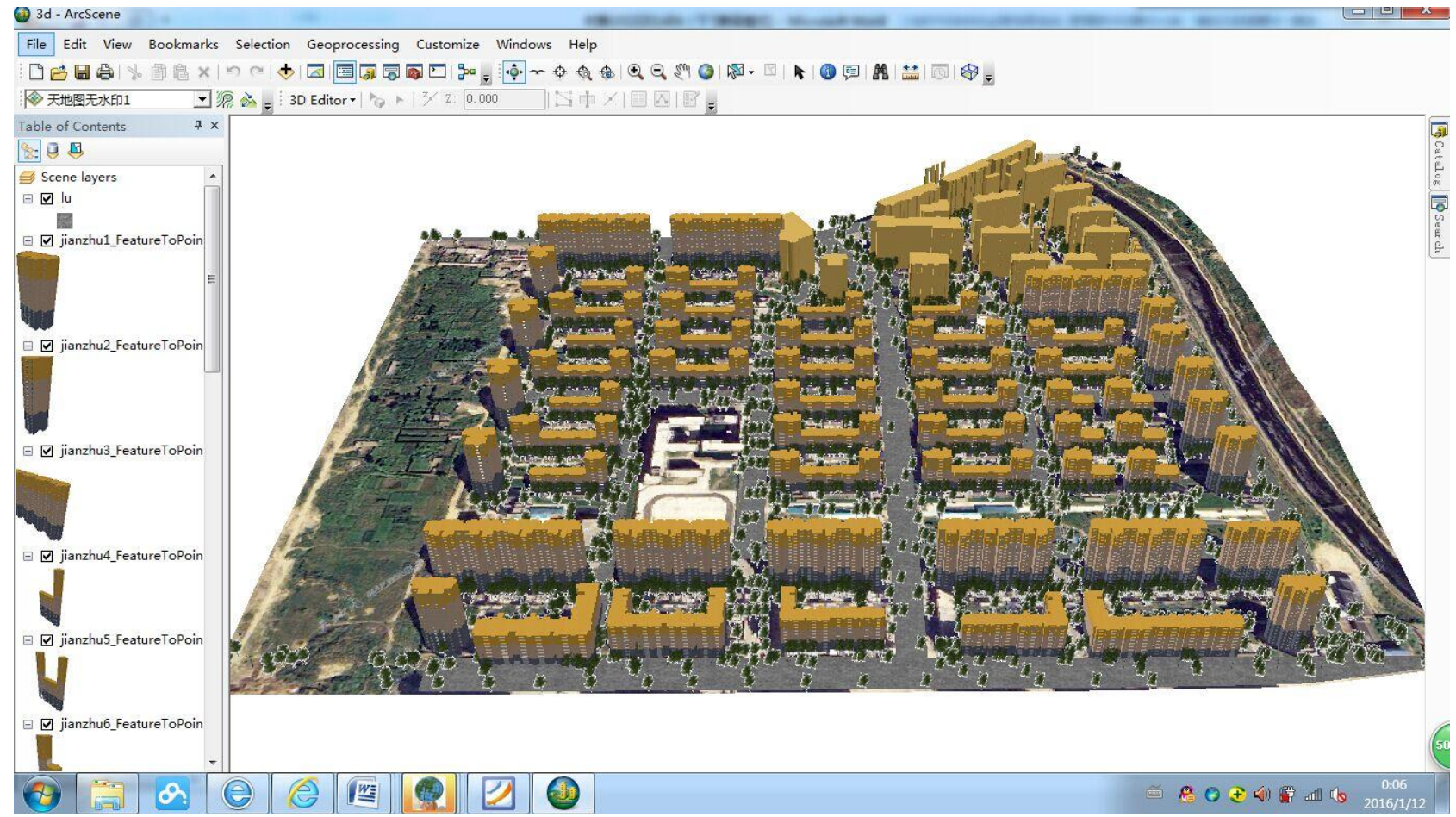

Fig. 5. Community 3D landscape.

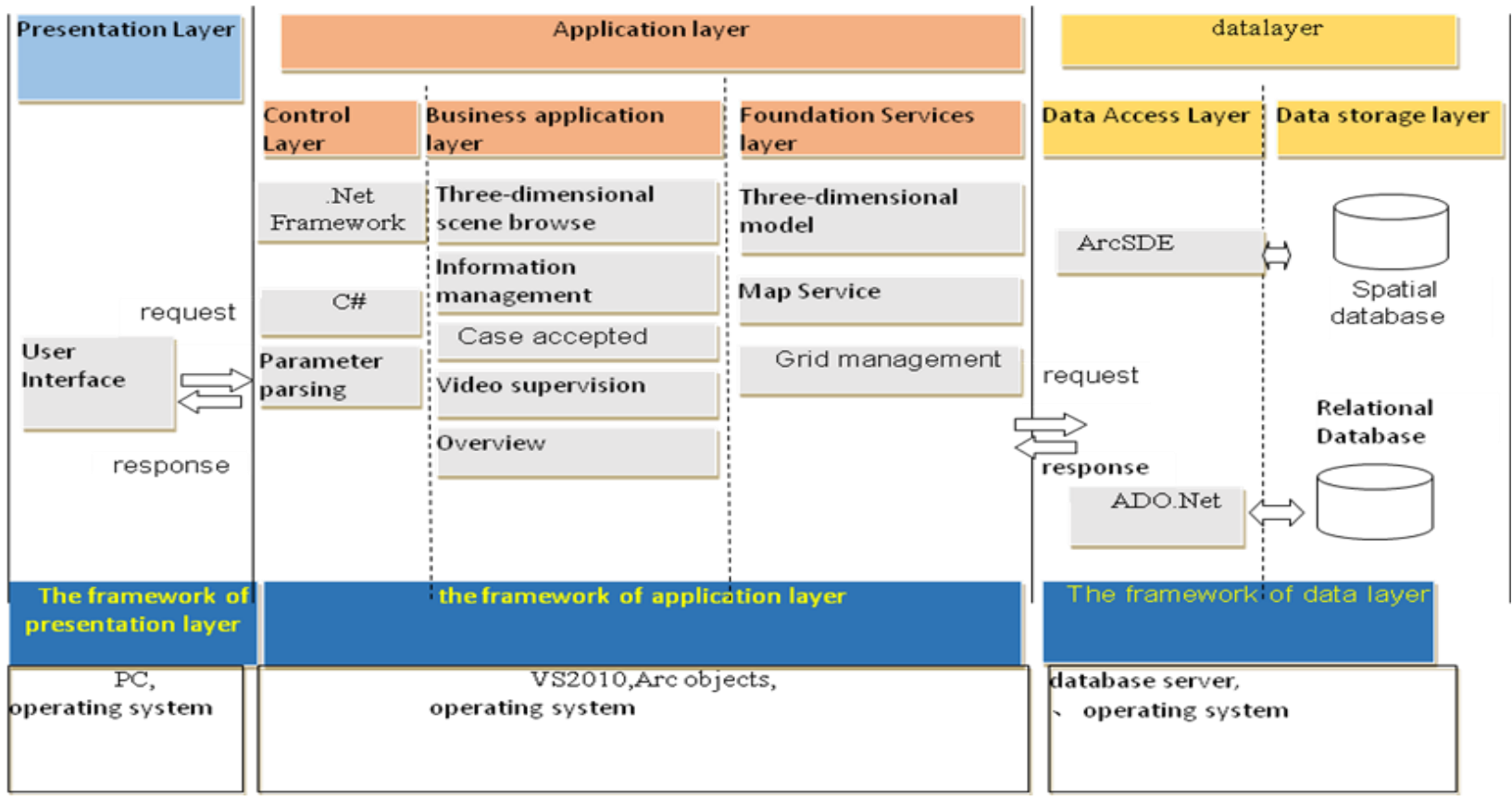

Fig. 6. System architecture diagram.

2) Application layer: application layer includes three levels: basic service layer, business application layer and control layer.

(1) Basic service layer: this layer provides the implementation method and the key algorithm of each module 
of the system, and provides guarantee for the normal operation of other layers.

(2) Business application layer: the layer is the core and key of the whole system layer, and it is the main specific realization of each function module. The specific functions of each module refer to system function module design and the realization of specific functions of the system.

(3) Control layer: the work of the control layer is to receive a request from the user, to make a judgment to call after the business layer. The results will be forwarded to the view. This system uses the Framework.NET to carry out the system design. The user's requests are achieved through the various controls of the operating system framework.

3) Performance layer: this layer mainly refers to the user interface, it is the users' experience which is shown in front of the user, and it is a bridge between human and machine. Users make the system complete some functions through the interface.

\subsection{D Basic Operation Function}

3D basic operating functions are shown in the form of toolbars, include seven tools: zoom in, zoom out, pan, the full-size display, flight, move and recognition. These shortcut tools can help users to easily manipulate and navigate the 3D map, which can meet the different requirements of users. Map view operation function is realized mainly by instantiating ICommand interface for the corresponding 3D scene operation, then using CurrentTool of SceneControl to get or set the current operation status of scenes in the scene control. The functional interface and implementation is shown in Fig. 7.

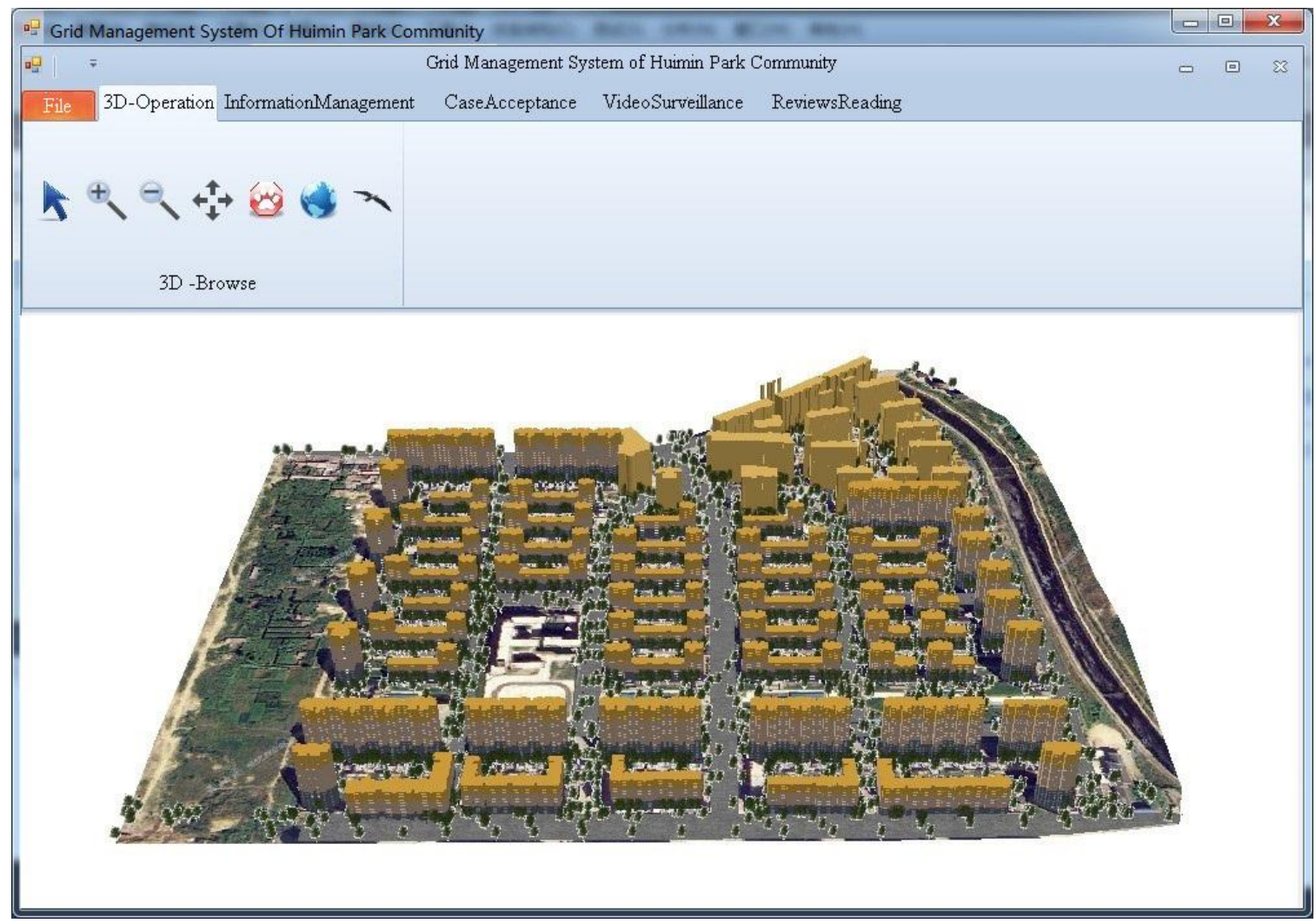

Fig. 7. 3D operation diagram.

\subsection{Information Management Function}

Daily inspection of grid managers is the first step of grid management which is a closed loop process of experimental community. The object of the grid patrol management is mainly the four types of grid, residential buildings, population, parts. In the inspection process, the information of the object can be queried. According to the actual appearance which is correspond directly to the three-dimensional model of the system, then click 
query with information, namely from the graphics to the property of the query, this operation can get residents reflect problems, such as a street lamp numbers problems, according to the number of query to the location of the street lamp is more convenient and quickly check the situation, which is from the property to the graphical query. In addition, grid manager found the actual situation of the management object occurrence changes or the original information is wrong after inspections, such as residential building tenants have move, the total number of the living has been changed, under these situation, we can edit the detailed information of the grid, for instance, the residential buildings, the population, the component, the edit tool includes adding, modifying, and deleting operations.

The information query function from graph to attribute is to choose the corresponding 3D object model in map can realize the inquiry of the detailed attribute information. This function combines the 3D model with the logical data, which makes the grid management personnel more vivid and specific to the spatial entity information. Specific method is first calling the method LocateMultiple of IScenegraph interface to complete the conversion which is from the screen coordinates where the mouse click on to 3D spatial coordinates, then using method Identify of class Campuse to get the 3D solid objects which the point correspond to and pass the entity object to display attribute information in detail.

1) Grid query. Users can click the grid in the 3D scene to show grid information window. The statistical information of six major categories "people, housing, places, things, events, organizations" of the grid will be shown in the window, including the total population number, storied building number, the total number of households, check number, the number of spare, brand building area, the number of elements such detailed information.

2) Residential building query. As shown above, the user can make the residents building information window show by clicking the three-dimensional model of residential buildings in the three-dimensional landscape. The detailed information of the residents building will be displayed in the window.

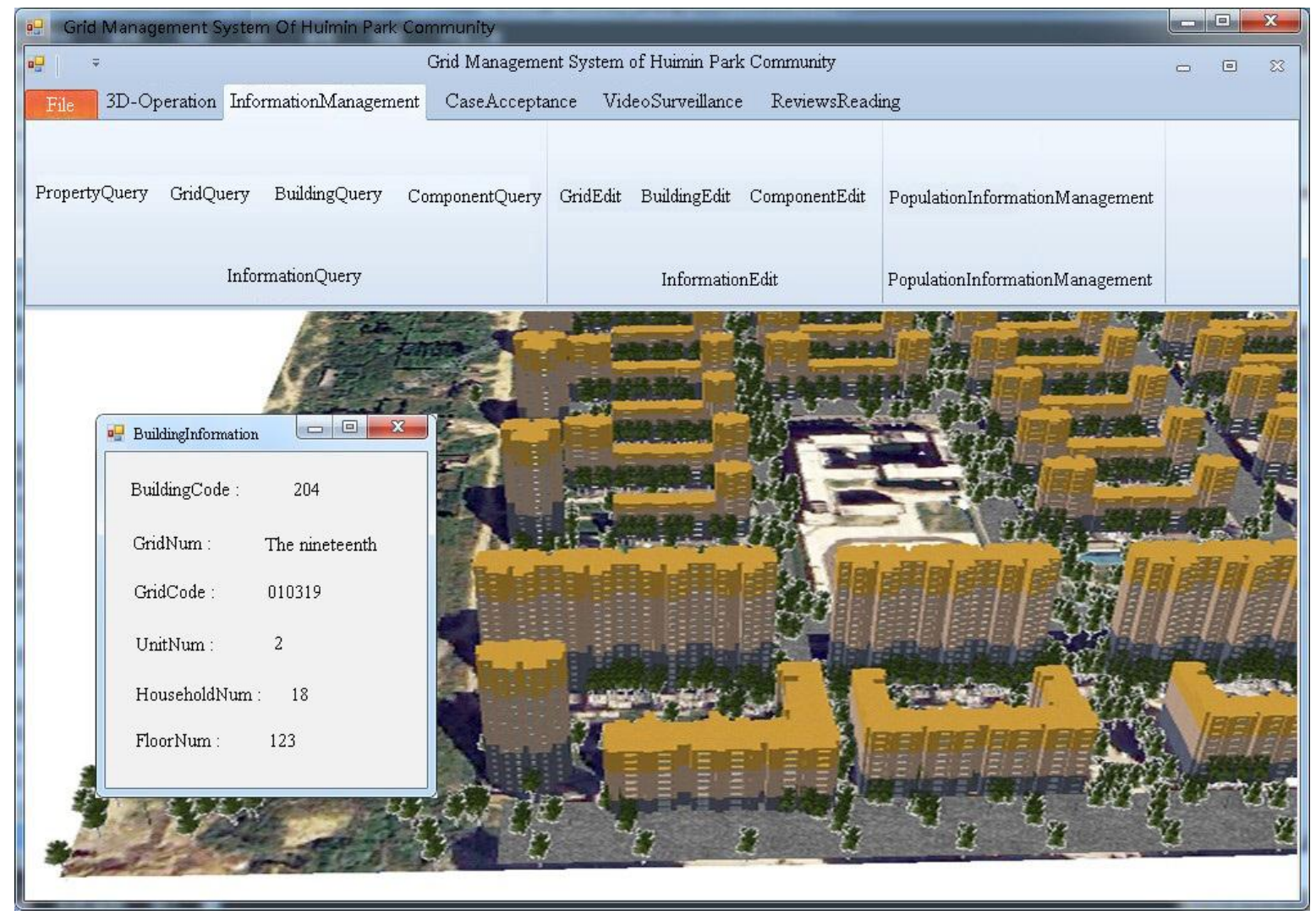

Fig. 8. Query residential building information.

3) Component information query. "Object" is the popular view of the components of the community. The 
"thing" in the six categories of information management is the management of components information. Grid managers need conduct regular inspections of components in the grid to find the problem. In the process of inspection, the basic information can be directly queried by clicking the component in the three-dimensional scene.

The information query function which is from the attributes to the graphics, that is user can set query conditions to find the corresponding graphics and highlight on the map through the attributes of grid, residential buildings or components. Specific implementation method is using the interface IQueryFilter to set the query conditions, and using the search method of IFeatureLayer.IFeatureClass to query the entity object, using the cursor object IfeatureCursor to express the results of the query and finally using method IActiveView.PartialRefresh to refresh the choice to make the query object highlighting.

Querying and positioning the three-dimensional models of grid, the residents building, the components according their attributes is realized as follows: click the corresponding " grid query "," residential building query", "the component query" button of "attribute query graphics" tab, then the corresponds query window will be shown, select the query field through property drop-down box, click the "field value "button, all the field value of the grid, residential buildings or components will be displayed in the list box, select the field values to determine the specific query object, click on" location "button to highlight 3D model.

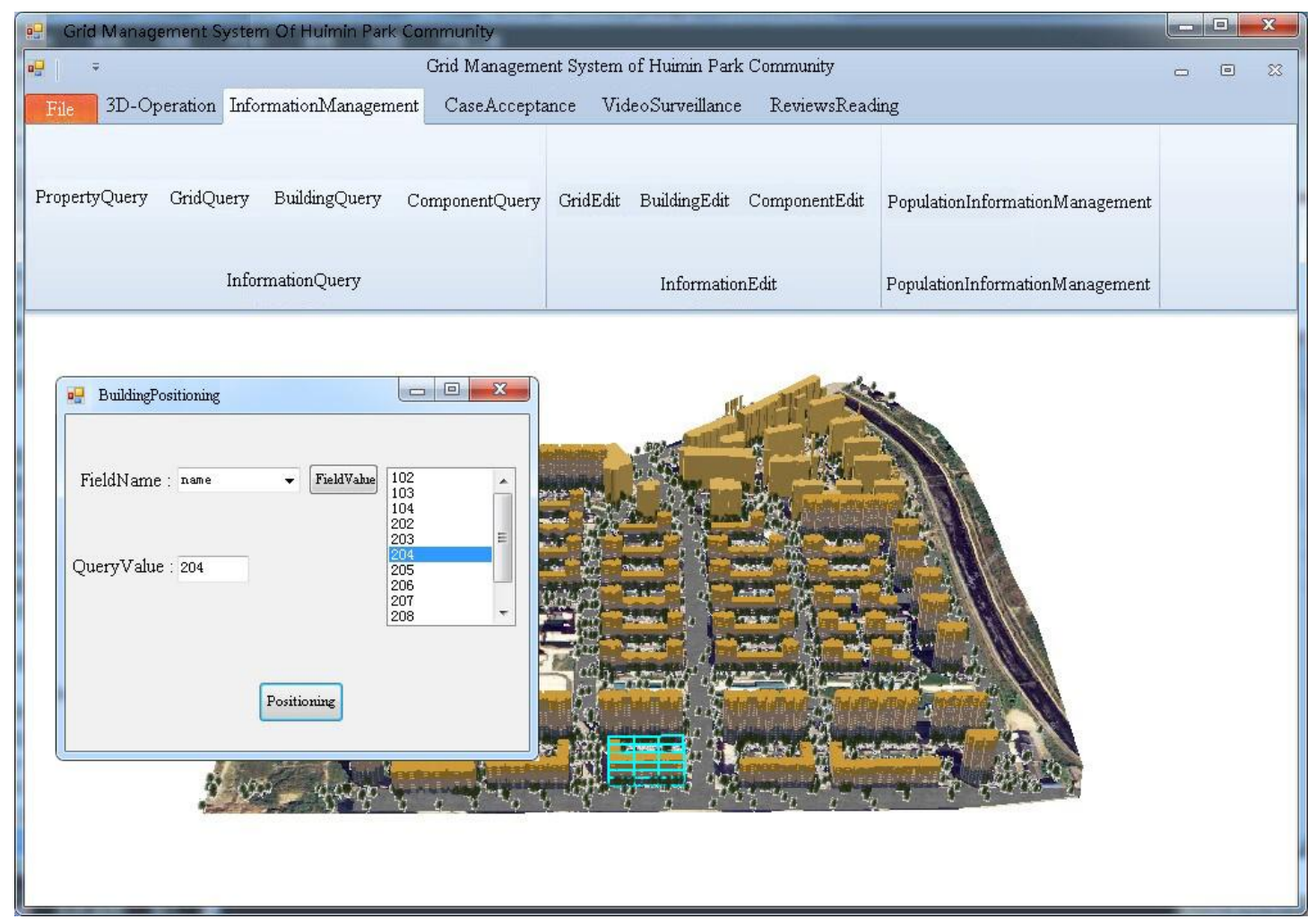

Fig. 9. Residential building locating.

\section{Conclusion}

The proposal of the grid community management model makes us realize that the old management model has been unable to adapt to the rapid development of the information age. With the development of GIS technology, the three-dimensional grid community management has become an important development direction.

1) It is feasible to use the GIS technology, grid management theory, and database technology to develop the grid management system.

2) Using three-dimensional modeling software 3DMAX and GIS software to build the 3D visualization environment of community, realizes the three dimensional browsing and operation, can enable the user to operate in a 3D environment, which can make up for the limitations of the two-dimensional graphics 
management model.

3) Based on 3D community environment, the realization of community grid management system can make community management intuitive, make the process of community grid management informationalized, and effectively improve the community management efficiency, so as to improve the community management and services to residents.

\section{Acknowledgements}

My deepest gratitude goes first and foremost to the professors and teachers at the department of the NASG Key Laboratory of Land Environment and Disaster Monitoring who supported this paper's work.

\section{Reference}

[1] Wang, K. F. (2014). Research on the community management ways based on three-dimensional digital community information system. Bulletin of Surveying and Mapping.

[2] Su, T. (2004). The Application of J2EE/XML in Digital Community Development, South west Jiaotong University.

[3] Li, M. (2014). The experience reference and revelation of the foreign urban community management system. Higher Education of Social Science.

[4] Weng, J. N., Wang, Ying, J., \& Cai, H. (2010). Constructing a core framework of visual engine for digital earth system. Science China Technological Sciences, 53.

[5] Bao, G. Z. (2012). Digital Community Information Systems Resarch Based On GIS Technology. Xi'an University of Science and Technology.

[6] Zhao, S., Ji, Y. Z., \& li, W. (2012). The practice and thoughts community GIS management system. China Resources Comprehensive Utilization, 30, 46-48.

[7] Yen, J. (2000). Special issue of decision supports systems-intelligent agents and digital community. Decision Support Systems, 28.

[8] Sillence, E., \& Baber, C. (2003). Integrated digital communities: combining web-based interaction with text messaging to develop a system for encouraging group communication and competition. Interacting with Computers, 16.

[9] Li, H. T., Ma, J. F., \& Fu, S. (2013). An anonymous data collection framework for digital community. Wuhan University Journal of Natural Sciences, 18.

[10] Bai, Y. J., \& Lv, D. J. (2010). Discussion of Urban Meters Unit Meshing Method. Jiangxi Surveying and Mapping, 1.

[11] Wang, C. J. (2014). Promoting City Management in the Age Of big Data: Problems and Solutions. Soochow University.

[12] Xu, W. P., Zhu, Q., Du, Z. Q., et al. (2010). Design and implementation of 3D model database for general-purpose 3D GIS. Geo-spatial Information Science, 13.

[13] Stephan, N., Andreas, B., \& Beat, F. et al. (2009). From historical city models to interactive 3D GIS requirements and approaches using the example of the city of solothurn. PFG Photogrammetrie.

[14] Reddy, S. K. (2015). A comparative study of grid management information server framework with MDS, RGMA, hawkeye and grid bus broker. International Journal of Computer and Communication System Engineering.

[15] Costa, M. (2015) Community Energy Storage Sizing for Grid Management Optimization. Anno accademico.

[16] Zhao, Q. (2015). How institutional pressures affect innovation diffusion in local government public services: The case of grid-based city management. Journal of Public Administration, 3(8). 
Yajing Liu was bron in Hebei China, in 1977. She graduated from China University of Mining and Technology (Beijing) in 2007. She received her doctorate degree in cartography and geographic information engineering.

She worked at the North China University Science \& Technology from 2007 till now. She teaches the professional courses in the mayor of geographic information science. 